\title{
WIRELESS SENSOR NETWORKS APPLICATIONS
} IN AIRCRAFT STRUCTURAL HEALTH MONITORING

\author{
Dragoljub Vujić* \\ Military Technical Institute, Belgrade, Serbia
}

\begin{abstract}
Aircraft industry has to meet a challenge of reducing operational and maintenance costs. One of the possible ways for reducing these costs is the introduction of wireless sensor networks (WSNs). WSNs are already finding a variety of applications for both safety-critical and non-safety critical distributed systems. This paper deals with the application of WSNs for aircraft structural health monitoring. Special attention has been given to the WSNs design issues using available components on the market. A general scheme for aircraft structural health monitoring using MicroStrain components has been proposed.
\end{abstract}

Key words: Wireless sensor networks, Aircraft structural health monitoring, Micro-electro-mechanical systems, Condition-based maintenance, Sensor node, Wireless communication

\section{INTRODUCTION}

Over the last decade Wireless Sensor Networks (WSNs) have been successfully applied in many engineering fields such as: structural health monitoring, industrial applications, environmental monitoring, traffic controls, health applications, etc. This paper deals with the application of WSNs for aircraft structural health monitoring. Generally speaking, the aim of structural health monitoring (SHM) is to monitor structures using embedded or attached non-destructive evaluation sensors and to utilize the data in order to assess the state of the structure. Often structures equipped with various types of sensors are compared to human nervous system. This means that SHM is the imitation of the human nervous system.

SHM is a new and improved way to make a non-destructive evaluation with a minimum of manual intervention. It includes all monitoring aspects which are related to damages, loads and conditions, which have a direct influence on the structure. Knowing the integrity of in-service structures on a continuous real-time basis is a very important objective for manufacturers, endusers and maintenance teams. Structural health monitoring allows an optimal use of the structure, a minimized downtime, and the avoidance of catastrophic failures. Therefore, structural health monitoring drastically changes the work organization of maintenance services: by aiming to replace scheduled and periodic maintenance inspection with condition-based maintenance and by drastically minimizing the human involvement, and thus improving safety and reliability [14].

Traditionally, the sensors deployed on the structure are connected through coaxial wires. However, cabling implies high installation and maintenance costs. Moreover, cables are subjected to wear or breakage. WSNs consistently reduce the installation and maintenance costs. Furthermore, the compact size and low cost of a single wireless sensor node enables the deployment of a large number of units on the monitored structure, especially in those locations difficult to be reached by wires, increasing the screening resolution of the system [04]. WSNs for SHM are supposed to operate for an extended period of time, e.g. few years, requiring minimal maintenance. For this purpose, the creation of a wireless sensing platform capable of collecting high-quality data requires a consistent engineering effort.

In the commercial and military aircraft there are a number of safety-critical and non-safety critical systems. These systems are based on wired connections and, therefore, they are complex and difficult to route. The Airbus A380, for instance, has over 300 miles of cables consisting of approximately 98.000 wires and 40.000 connectors [20]. Cable routing is quite a complex task, as for example, the power cable and electrical signal cable should be physically separated to avoid electrical interference. Also, harsh environmental conditions impose physical restrictions on the use of a wire harness. Replacement of the current wire harness-based sensors with a wireless 
sensor network (WSN) can help to achieve the goal of increasing the number of sensors, as well as, the system redundancy. It will also reduce the aircraft system weight and lead to improved fuel efficiency and reduced carbon emissions. The European goals for 2020, for instance, include a $50 \%$ cut in $\mathrm{CO} 2$ emissions. Replacing the physical cabling by wireless connections also offers significant benefits as regards flexibility, interoperability, mass reduction and improved robustness. Use of WSN also enables reduction in direct costs and maintenance costs.

\section{WIRELESS SENSOR NETWORKS TECHNOLOGY}

Wireless network refers to any type of computer network which is not connected by cables. It is a method by which homes, telecommunications networks and business installations avoid the costly process of introducing cables into a building, or as a connection between various equipment locations. Wireless telecommunications networks are generally implemented and admin- istered by using a transmission system called radio waves. AM radio, FM radio, satellite radio, satellite TV, satellite Internet access and broadcast TV are, in fact, wireless networks. Hence, the usage of wireless technology is very convenient [13].

WSNs consist of spatially distributed autonomous sensors designed to monitor physical parameters or environmental conditions, such as temperature, strain, pressure, vibration, sound, motion, pollutions, etc. Consequently, the sensors cooperatively pass their data through the network to a main location. The base station may communicate with the user or task manager node via Internet or Satellite.

A wireless sensor, also known as a mote (reMOTE), smart dust, smart sensor or sensor node within the network performs the function of sensing, data processing and wireless data transmission. It is powered by an individual power source which often consists of a battery with a limited energy budget. The general scheme of WSNs is presented on Figure 1.

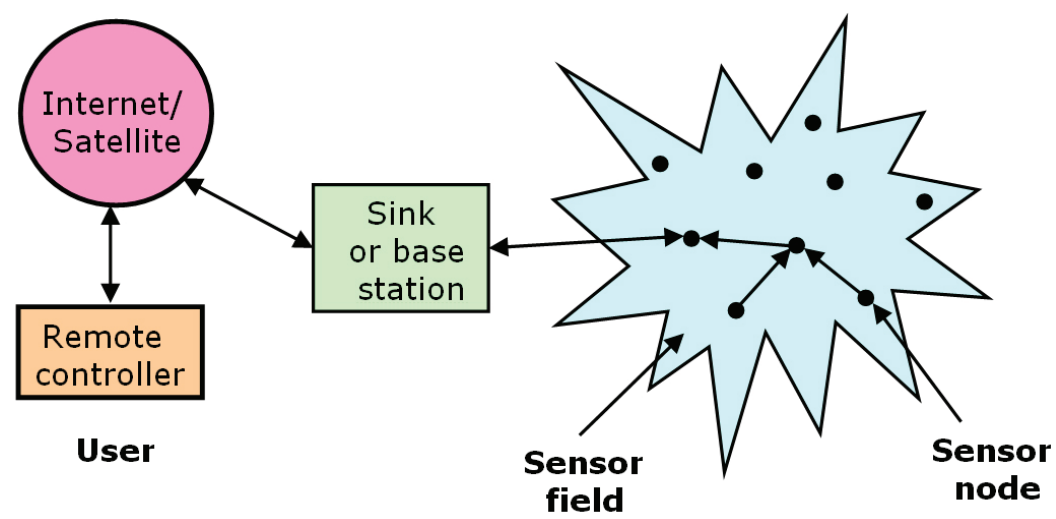

Figure 1: A general scheme of wireless sensor networks

The development of WSNs largely depends on the availability of low-cost and low-power hardware and software platforms for sensor networks. With the micro-electro-mechanical system (MEMS) technology, the size and cost of a sensor node have been significantly reduced. This is to say, energy efficiency can significantly be enhanced if energy awareness is incorporated in the design of system software, including the operating system, and application and network protocols. System lifetime can considerably be prolonged by incorporation energy awareness into task scheduling process [13].

The nodes communicate wirelessly and often self-organize after being deployed in ad hoc fashion. Systems of 1000 s or even 10.000 nodes are anticipated. Such systems can revolutionize the way we live and work. Currently, WSNs are beginning to be deployed at an accelerated pace. It is not unreasonable to expect that in 10-15 years that the world will be covered with wireless networks with assess to them via the Internet. This can be considered as the Internet becoming a physical network [10].

\section{Sensor node architecture}

A sensor node typically consists of five main components (Figure 2): one or more sensors gather data from the environment and report the data to the microprocessor. A microprocessor is a central part of a wireless sensor node. It processes all the data that receives from memory, 
sensor, or transceiver. A transceiver communicates with the environment. It is used radio frequency (RF) as a transmission medium to send data wirelessly. The transceiver can take data from a microprocessor to send it over the air and vice versa. A memory is the main resource for storing programmes and intermediate data coming from the sensors or the transceiver. The size of the memory depends on the application of the sensor. The battery supplies all parts with energy. To assure a sufficiently long network lifetime, energy efficiency in all parts of the network is crucial. Although most sensors have a traditional battery, there is an early stage research regarding production of sensors without batteries, using similar technologies applied to pas- sive radio frequency identification (RFID) chips without batteries. The sensor nodes are usually scattered in a sensor field. Each of them has the capabilities to collect data and route data back to the base station. The base station may communicate with the task manager node via Internet or Satellite. In [06] the node deployment models in WSNs have been explored. Various architectures and node deployment strategies have been developed for WSN, depending upon the requirements of application. The authors focused on five deployment schemes for sensor networks environments, random deployment, grid deployment, group-based deployment, and grid-group deployment.

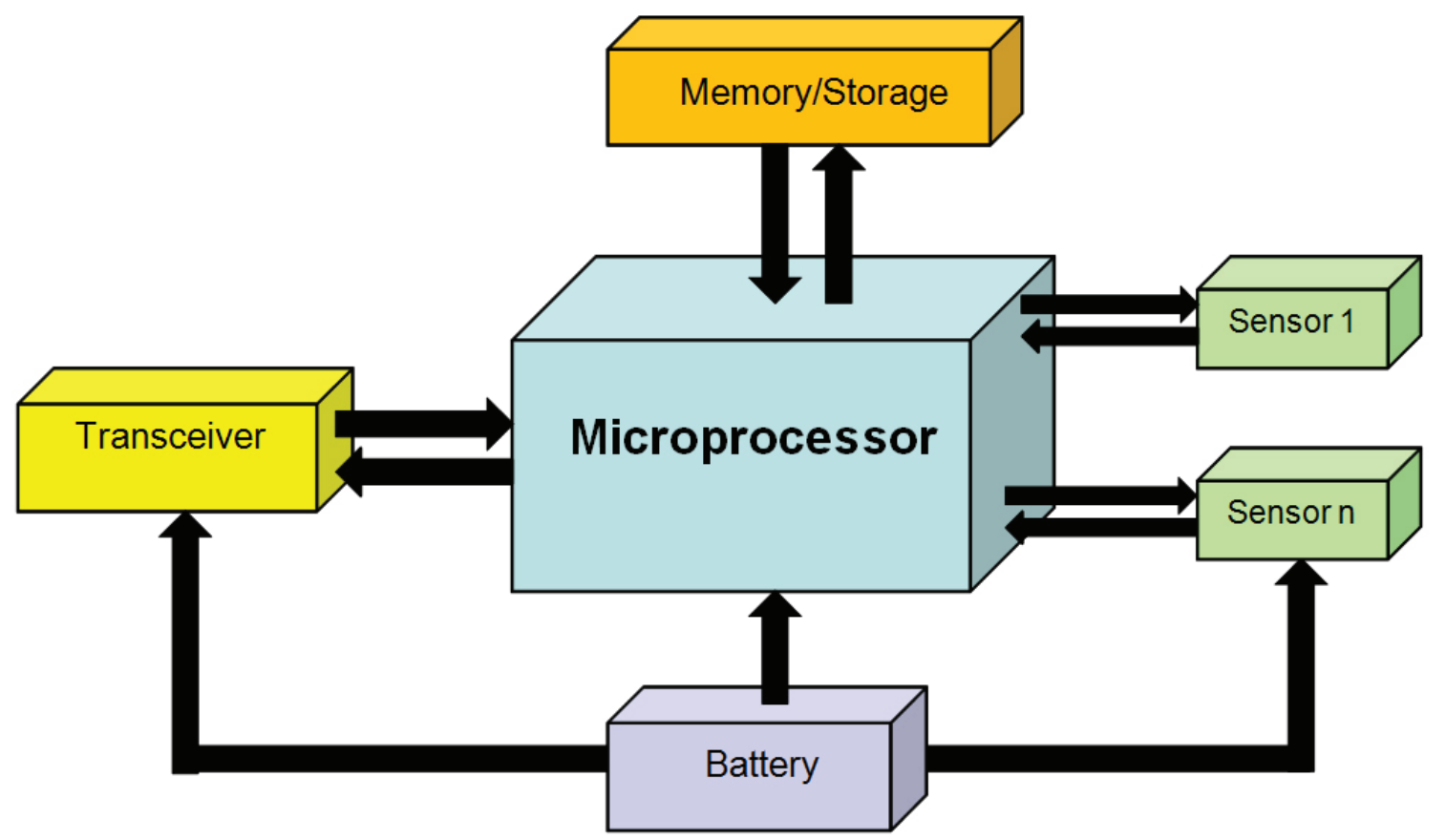

Figure 2: Hardware components of a sensor node

\section{Energy efficiency in routing}

A number of research papers have already been accomplished in routing in WSN, since energy efficiency is more important for wireless sensor networks than any other networks. In wireless communication, data transmission consumes more power than data processing. The battery power of the node will be reduced whenever they transmit a great number of data proportionately. In order to reduce the data size we can prefer techniques like data fusion or aggregation. Data fusion is that in which the sensed data are fused at a certain point for transmitting them at a reduced size. However, there is a problem, showing of lack in precision and accuracy of data from various sensor nodes [07]. In order to prolong the lifetime of the WSN, designing efficient routing protocols appear to be critical. It has been established that most of the energy consumption in a WSN comes from data reception and transmission. Therefore, a good routing protocol can reduce the number, as well as, the size of the unnecessary transmissions which take place. Thus, the routing protocol helping to alleviate the energy crisis in WSNs. Hierarchical routing algorithms are techniques with special advantages related to scalability and efficient communication. The main aim of hierarchical routing is to optimize energy consumption of sensor nodes by arranging the nodes into clusters [07]. Data aggregation and fusion is performed within the cluster in order to decrease the number of transmitted messages. 


\section{WIRELESS SENSOR NETWORKS IN AIRCRAFT STRUCTURAL HEALTH MONITORING}

There are a number of WSNs applications in structural health monitoring of aircraft. Here, it will be briefly presented some of them.

Researchers from the research institute of China have applied a wireless sensor network for static testing of a real aircraft undercarriage [15]. The developed wireless sensor network system consisted of 14 sensor nodes and 4 cluster heads. The authors concluded that the system design may be much more complicated when the number of testing points which have to be measured will greatly increase. Furthermore, fatigue testing for full-scale structure requires higher data transmission rates, data synchronization and data buffer processing capacity. Therefore, hardware capabilities for the WSN based aircraft strength testing systems should be improved in further research. Networking and routing protocols should be seriously studied in order to solve these problems.

Wireless sensor modules were integrated into the pitch link of a Bell M412 helicopter [01]. Pitch link loads were recorded and periodically transmitted into the cabin during flight. Wireless sensors included strain gauges, accelerometers and thermocouples. Hard-wired sensors included gyroscopes, accelerometers and magnetometers. Wireless technologies for tracking the load history of helicopter rotating components, combined with inertial and global positioning system (GPS) information, can be used to compute structural loads with improved accuracy. The integration of these sensor systems will lead to reduced cost flight testing, improved safety, and enhanced condition - based maintenance.

In [02] the first flight tests of a synchronized wireless structural monitoring system aboard a helicopter has been presented. Combined with energy harvesters, these new wireless sensing networks can be deeply embedded into structures and structural components for improved condition - based maintenance and advanced structural health and usage management systems.

Because of the increasing use of composite materials for aircraft structures, it is necessary to develop new methods for aircraft structural health monitoring. Most of the failures of the laminated composite structures originate from delamination of layers. As regards metal aircraft structures, cracks develop and eventually lead to failures. In both of these cases, visual inspection is not a reliable method for failure detection. This calls for a vibration analysis-based on failure detection method. Currently scheduled aircraft structure maintenance methods have a high maintenance cost. Several studies have been conducted to develop health monitoring algorithms which use the data from strain sensors embedded into the composite structure. WSN can be embedded into the composite structure which will harvest the vibration energy and will transmit the realtime data to the central health monitoring unit. These sensors will be used to monitor the internal parameters like cracks, strain, as well as, external parameters such as temperature, load, etc. Because of this, the use of WSN, powered by energy harvesting techniques will increase the number of sensors and their lives. Hence, the real-time data will enable the use of condition-based maintenance, thereby preventing catastrophic failure of aircraft structures. Although the use of MEMS is one of the promising technologies for implementation of WSN-based aircraft structural monitoring, optimum energy harvesting and power management methods for MEMS sensors have to be further improved. The integration of sensors and airframe has to be studied, as well, the effect on the structural strength of composite materials due to embedded sensors.

\section{WIRELESS SENSOR NETWORKS DESIGN ISSUES USING AVAILABLE COMPONENTS ON THE MARKET}

A well known USA company MicroStrain has deployed wireless sensors and wireless sensor gateways for a number of applications. Sensors that measure strain, acceleration, displacement, pressure, temperature, inertial loads, and torques have been combined in time synchronized networks to provide a rich amount of information for improved condition based maintenance. Sensors can be quickly deployed in discrete locations of the aircraft structure. Wireless sensing systems are ideal for both small scale applications requiring a few sensor nodes and large scale applications requiring hundreds of sensor nodes. With highly synchronized data sampling, and extended range communication, MicroStrains's WSNs are able to collect and aggregate data in a single database, and push it to the cloud for remote ac- 
cess. Wireless gateways, or base stations, provide seamless communication between a host PC, single board computer or microcontroller, and remote wireless sensor nodes.

MicroStrain's Lossless Extended Range Synchronized (LXRS) wireless sensor networks are scalable, fast, and $100 \%$ reliable under most operating conditions. The main features of the LXRS wireless sensor systems are as follows:

- Lossless wireless communications protocols provide $100 \%$ packet success rate;

- Extended Range radio link to 2 kilometers;

- Scalable wireless sensor networks support continues, burst, and hybrid sampling modes;

- Time Synchronized to +/-32 microseconds.

The LXRS Wireless Sensing System works by leveraging advanced bi-directional radio communications protocols. When data are received without errors by the wireless sensor data aggregator (WSDA) base station, the WSDA sends an acknowledgement that these packets were received. Data that are not acknowledged remain within each LXRS sensor node's non-volatile memory for re-transmission according to the network scheduler. It has to be noted that data are time-stamped by each node at the time of analog-to-digital (A/D) conversion. Therefore, even when re-transmitted, all data are accurately time stamped.

\section{Wireless accelerometer node}

Wireless Accelerometer Node (The G-Link LXRS) presented in Figure 3 features on-board triaxial $\pm 2 \mathrm{~g}$ or $\pm 10 \mathrm{~g}$ MEMS accelerometers and an internal temperature sensor. G-Link LXRS can be employed to measure vibration or acceleration, or as a tilt sensor or inclinometer. The node can simultaneously log data to internal memory and/or transmit real-time data to a host computer at user programmable data rates up to $4096 \mathrm{~Hz}$. Its form factor allows remote, long term deployment. Node Commander software supports configuration of the wireless node including discovery, initialization, radio frequency, sample rate, reading/writing to node EEPROM, calibrating node sensors, managing node batteries including sleep, wake, and cycle power, and upgrading node firmware. The G-Link -LXRS is compatible with any WSDA - Base, WSDA - 1000 or SensorCloud. At the heart of MicroStrain's LXRS Lossless Data Wireless Sensor Networks are WSDA (Wireless Sensor Data Aggregator) gateways, which use exclusive beaconing protocols to synchronize precision timekeepers within each sensor node in the network. The WSDA also coordinates data collection from all sensor nodes. Users can easily program each node on the scalable network for simultaneous periodic, burst, or data logging mode sampling with Node Commander software, which automatically configures radio communication to maximize the aggregate sample rate. Optional SensorCloud enabled WSDA support autonomous web-based data aggregation.

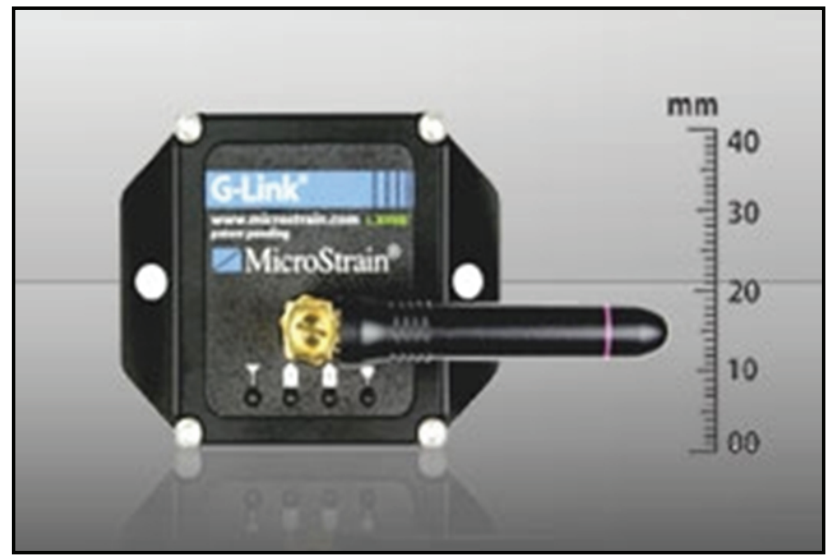

Figure 3: Wireless Accelerometer Node

At the heart of MicroStrain's LXRS Lossless Data Wireless Sensor Networks are WSDA (Wireless Sensor Data Aggregator) gateways, which use exclusive beaconing protocols to synchronize precision timekeepers within each sensor node in the network. The WSDA also coordinates data collection from all sensor nodes. Users can easily program each node on the scalable network for simultaneous periodic, burst, or data logging mode sampling with Node Commander software, which automatically configures radio communication to maximize the aggregate sample rate. Optional SensorCloud enabled WSDA support autonomous web-based data aggregation.

\section{The WSDA - 1000 Wireless Sensor Data Aggregator}

The Wireless Sensor Data Aggregator (WSDA 1000 ) presented in the Figure 4 is a single-board computer with Ethernet connectivity designed to operate as an integral part of MicroStrain LXRS Wireless Sensor Networks. The WSDA - 1000 is capable of collecting lossless data from a wide range of MicroStrain wireless sensor nodes operating in LDC or Synchronized sampling mode. The wirelees sensor network can be set up and 
controlled remotely via LiveConnect and NodeCommander. Once set up, the WSDA - 1000 may operate in one of three distinct modes: SensorCloud Enabled, LAN, and Standalone.

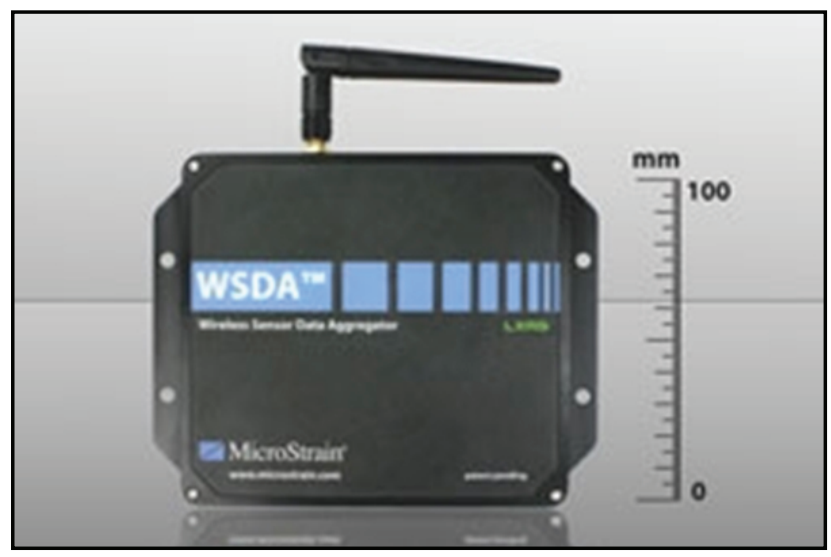

Figure 4: WSDA (Wireless sensor Data Aggregator) - 1000

The general features and benefits of the WSDA - 1000 Wireless Sensor Data Aggregator are the following:

- Lossless wireless communication protocol provides $100 \%$ packet success rate;

- Programmable communication range from $70 \mathrm{~m}$ to $2.000 \mathrm{~m}$;

- Scalable wireless sensor networks supporting continuous, burst, and hybrid sampling modes;

- Time synchronized to +/- 32 microseconds;

- Minimal setup required; collect data within minutes;

- Autonomously aggregates wireless sensor data;

- Supports a wide range of MicroStrain wireless sensor nodes transmitting in LDC or Synchronized Sampling modes;

- 2 GB non-volatile embedded flash for local storage;

- Command, control, and monitoring of a remote wireless sensor network from user PC;

- Web interface for system configuration;

- Full industrial temperature range supported $\left(-40^{\circ} \mathrm{C}\right.$ to $\left.85^{\circ} \mathrm{C}\right)$;

- Seamlessly integrates with SensorCloud for world-wide data access and visualization;

- Includes a free basic SensorCloud account;

- Local storage is used for SensorCloud caching.

\section{Sensor Cloud}

Data stored on SensorCloud represents discrete sensor values stored as a function of time. MicroStrain's customers may want to create mathematical expressions based on one, or a combination of these sensed values. In order to support customer's unuque requirements, MicroStrain has developed MathEngine, which enables users to upload algorithms and the output of these algorithms can be represented as an additional sensor channel or "virtual” sensor. For example, one can use strain data to create an estimate of fatigue using a rainflow algorithm. When the fatigue rate is high, an alert can be generated by Sensor Cloud. In another example, vibration data collected over time can be converted into the frequency domain using FFT and analyzed to detect anomalies.

MicroStrain wireless sensor nodes can easy connect to SensorCloud. All that it needs is a MicroStrain WSDA Wireless Sensor Data Aggregator and an internet-enabled network connection. SensorCloud supports a broad range of wireless sensor sample rates, ranging from one sample per hour to 100.000 samples per second.

SensorCloud 's Live Connect feature can be used to establish a direct connection to MicroStrain wireless sensor network, from anywhere in the world. Once connected, user can access the full range of sensor network advanced features using Node Commander PC software, including:

- View high speed streaming data in real-time;

- Trigger and download datalogging sessions;

- Change network and node configuration settings;

- Update wireless sensor node firmware overthe-air.

The coupling of advanced wireless sensor networks with innovative cloud-based data analytics revolutionizes performance monitoring of remote structures. Used over the long-term, operators can gain valuable insight into the deterioration of structures and its corresponding effect on performance. The general scheme of the MicroStrain's Wireless Sensor Network is presented in the Figure 5 . 


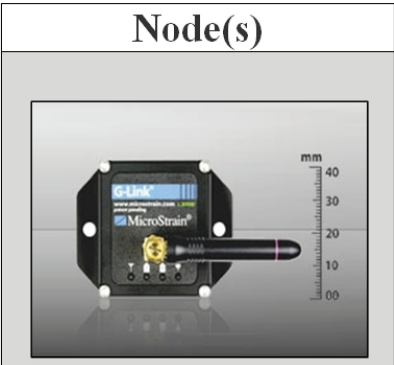

Wireless Accelerometer Node

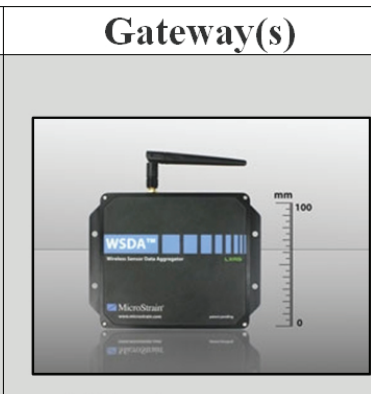

Wireless sensor Data Aggregator
Interface

Sensor Cloud
Data Access

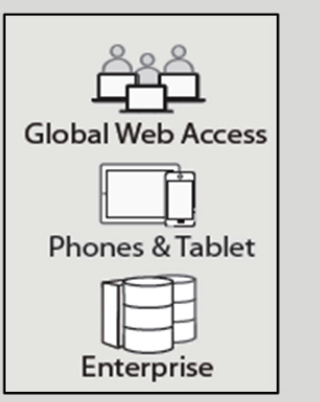

Figure 5: General scheme of the MicroStrain's Wireless Sensor Network

\section{CONCLUSION}

The aircraft industry will greatly benefit from the use of WSNs. These benefits through weight savings, reduction in subsystems design complexity and improved condition based maintenance will directly benefit the airlines in terms of additional revenues, as well as, lower operational and maintenance costs. Nevertheless, using wireless technology gives the potential to lead to more efficient future aircraft designs and quicker time-to-market.

The presented general scheme of the Wireless Sensor Network, based on some components available on the market, can be applied for aircraft structural health monitoring. The user has to choose the type of sensors which wants to apply. Sensors can be quickly deployed in discrete locations of the aircraft structure. As mentioned, the MicroStrains's sensing systems are ideal for both small scale applications requiring a few sensor nodes and large scale applications requiring hundreds of sensor nodes. Wireless sensor nodes are able to collect and aggregate data in a single database, and push it to the cloud for remote access.

\section{REFERENCES}

1) Arms, S. W.,a Townsend, C. P., Churchill, D.L., Galbreath Jian, J. H., Corneau B., Ketcham R. P., Phan N. (2008): Energy Harvesting, Wireless, Structural Health Monitoring and Reporting System, 2nd Asia-Pacific Workshop on SHM, Melburn

2) Arms, W. S., Townsend, P.C., Galbreath, H. J., Distasi1, J. S, Liebschutz, D., Phan, N. (2011): Flight Testing of Wireless Sensing Networks for Rotorcraft Structural Health and Usage Management Systems, AIAC14 Fourteenth Australian Congress, Melbourne, Australia

3) Boano, A. C. (2009): Application Support Design for Wireless Sensor Networks, Master Thesis, School of Information and Communication Technology, Kungliga Tekniska Högskolan.

4) Bocca, M. (2011): Application - Driven Data Processing in Wireless Sensor Network, $\mathrm{PhD}$ Thesis, The Aalto University School of Electrical Engineering, Finland

5) Bokare, M., Ralegaonkar, A. (2012): Wireless Sensor Network: A Promising Approach for Distributed Sensing Tasks, Excel Journal of Engineering Technology and Management Science, ISSN2249-9032, Vol.I, No 1.

6) Chandrasekaran, V., Chanmugam A. (2012): A Review on Hierarchical Cluster Based Routing in Wireless Sensor Networks, Journal of Global Research in Computer Science, ISSN-2229-371X, Volume 3, No 2

7) Jian, Wu., Shenfang, Y., Genyuan, Z., Sai, J., Zilong, W., Yang, W. (2009): Design and Evaluation of a Wireless Sensor Network Based Aircraft Strength Testing System, Sensors, 9, 4195-4210, ISSN 1424-8220

8) Mohan, L., Ranjitha, B., Begum S. A. (2012): Improving Performance in Wireless Sensor Networks Using MEMS Technology, International Journal of Electronics Communication and Computer Engineering, Volume 3, Issue (1) NCRTCST, ISSN 2249-071X.

9) Nordblom, T., Galbreath, J. (2012): Wireless Sensor Networks for Improved Long-Term Bridge Performance, MicroStrain, Inc., Long-Term Bridge Performance White Pape, Williston, VT 05495. 
10) Rao, G. S., Vallikumari, V. A. (2012): Beneficial Analysis of Node Deployment Schemes for Wireless Sensor Networks, International Journal of Advanced Smart Sensor Network Systems (IJASSN), Vol. 2, No 2.

11) Sampigethaya, K., Poovendran, R., Li, M., Bushnell, L., Robinson, R. (2008): Security of Wireless Sensor Network Enabled Health Monitoring for Future Airplanes, 26th International Congress of the Aeronautical Sciences ICAS 2008.

12) Singh, S. K., Singh, M. P., Singh, D. K. (2010): Applications, Classifications, and Selections of Energy-Efficient Routing Protocols for Wireless Sensor Networks, International journal of advanced engineering sciences and technologies, 1(2) 85-95.

13) Stankovic, A. J., (2006): Wireless Sensor Networks, Department of Computer Science, University of Virginia, Charlottesville, Virginia 22904.

14) Vujic, D. (2011): Recent trends in structural health monitoring of aircraft, 4th International scientific conference OTEH 2011, Proceedings, ISBN 978-86-81123-50-8, Military Technical Institute, Belgrade, pp. 153-158

15) Vujic, D. (2011): Structural Health Monitoring of Aircraft and Other Complex Structures, 2th DQM, International Conference, Life Cycle Cycle Engineering and Management, ICDQM-2011, Proceedings ISBN 978-8686355-06-5, Belgrade, Serbia.

16) Vujic, D.(2011): Some Methods For Aircraft Structural Health Monitoring, International
Conference, Maintenance and Production Engineering KODIP - 2011, Herzeg Novi, Montenegro.

17) Vujić, D., Stojić, R., Filipović, Z. (2012): Wireless Sensor Networks Technology in Aircraft Structural Health Monitoring, 5th International Conference OTEH 2012, Proceedings, ISBN 978-86-81123-58-4, Military Technical Institute, Belgrade.

18) Wagner, S. R. (2010): Standards-Based Wireless Sensor Networking Protocols for Spaceflight Applications, 2010 IEEE Aerospace Conference, NASA Johnson Space Center, 2101 NASA Parkway, Houston, Texas 77058

19) Yedavalli, R. K., Belapurkar R.K. (2011): Application of wireless sensor networks to aircraft control and health management systems, Journal Control Theory Application, 9(1) 2833, DOI10.1007/ s11768-011-0242-9

20) Yu, Y. (2010): Wireless sensor networks for health monitoring, Department of Electrical Engineering, Penn State University

21) Zhao, X., Qian, T., Mei, G., Kwan, C., Zane, R., Walsh, C., Paing, T., Popovic, Z. (2007): Active health monitoring of an aircraft wing with an embedded piezoelectric sensor/actuator network: II. Wireless approaches, Smart Mater. Struct., 16, 1218. doi:10.1088/09641726/16/4/033.

Paper sent to revision: 19.12.2014.

Paper ready for publication: 12.06.2015. 